(e.g. GCP) as well as more specialized topics (e.g. comparative effectiveness research). They can also be used to quickly respond to breaking issues (e.g. single IRB review). In an initial survey targeting University of Virginia clinical research coordinators, 54 (68\%) of 79 respondents reported having viewed a learning shot. Among those who had, 41 (84\%) of 49 respondents reported that learning shots were helpful to their learning needs. Continuous evaluation is expected to further inform how learning shots meet clinical and translational science education needs. DISCUSSION/SIGNIFICANCE OF IMPACT: Learning shots are an innovative and versatile educational tool for clinical and translational science that can be used to quickly and effectively convey important research information in response to an increasingly complex research environment and diverse learner needs.

\section{A Landscape Analysis of CTSA websites for Clinical Research Professional Training Opportunities}

Karen K Carter ${ }^{1}$, and Carolynn Thomas Jones ${ }^{1}$

${ }^{1}$ The Ohio State University

OBJECTIVES/GOALS: We conducted a review of CTSA websites to understand the current landscape for CRP institutional professional development and training revealed in the CTSA hub websites. METHODS/STUDY POPULATION: We accessed and reviewed 59 currently funded CTSA hub websites for evidence of CRP training opportunities. Parameters reviewed included: 1) opportunities were specified for CRPs versus $\mathrm{K}$ and $\mathrm{T}$ trainees; 2) mandated training; 3 ) leveling; 4) delivery methods/resources; 5) public accessibility; 6) unique features. The website reviews informed a REDCap survey sent to the CTSA Administrators $(\mathrm{n}=149)$ and the Coordinator Taskforce $(\mathrm{n}=105)$ listservs to gain additional knowledge of CRP training available at the institution. A subsequent repeat review of the CTSA hub websites will be conducted to determine evolving trends. RESULTS/ ANTICIPATED RESULTS: A total of 40 responded to the survey from 59 CTSA hubs. Survey results are being analyzed. Website review data are being tabulated and the subsequent review of websites will be collected in February. Those findings are pending and will include a comparison of prior findings. $42 \%$ of CRP hubs list CRP training within the CTSA hub website. Required onboarding training (beyond CITI certificates) is revealed for some hubs (15\%). DISCUSSION/ SIGNIFICANCE OF IMPACT: On our initial website review less than half of the CTSA hub websites list specific CRP training on their website. Many were hidden behind firewalls and could not be reviewed for content. The REDCap Survey will provide more granular descriptions of programs. Data from a second website review will be collected for comparison. Based on a preliminary re-review of sites, there is a suggestion of increasing CRP workforce development information. CTSAs are well-positioned to be a central hub for promoting educational excellence of the institutional workforce, for medical centers and in other venues where clinical research is performed.

A Telehealth Approach to Improving Healthcare to Rural and Underserved Populations

Erica Francis, Franc ${ }^{1}$, Jennifer Kraschnewski, Ruth Hogentogler, Kimberly Buckner, Jackie Sabol, and Kara Bowers

${ }^{1}$ Penn State Clinical and Translational Science Institute

OBJECTIVES/GOALS: Project Extension for Community Health Outcomes (ECHO), a telehealth model, was launched at Penn State
University in 2018 to connect specialists with community providers to provide education on best clinical practices. We aim to describe clinical topics covered and relevant provider level outcomes. METHODS/ STUDY POPULATION: The heart of the ECHO model is a hub-andspoke knowledge-sharing system. The ECHO model has four core principles: 1) use technology to leverage scarce resources; 2) share best practices to reduce disparities; 3 ) employ case-based learning to master complexity; 4) monitor outcomes to ensure benefit. Unlike telemedicine, where outside specialists assume the care of the patient, Project $\mathrm{ECHO}$ is a guided learning community aimed at practice improvement: providers receive mentoring and feedback on de-identified patient cases, strengthen their skillset, and retain responsibility for their patients. RESULTS/ANTICIPATED RESULTS: Clinical topics launched include Medication Assisted Treatment for Opioid Use Disorder, Ehlers Danlos Syndrome, Polyneuropathy, and Dementia. In addition, we launched a nutrition-focused ECHO with Boy Scout summer camp leaders in 26 states, reaching 107,347 scouts. Over the past year we have reached 118 clinicians in 62 clinics within 19 counties in Pennsylvania, providing a total of $268 \mathrm{CME}$ hours. These providers have treated 2,294 patients and reported increased knowledge (94\%), decreased sense of professional isolation (86\%), and improvement in ability to provide patient care (92\%) following completion of an ECHO series. DISCUSSION/SIGNIFICANCE OF IMPACT: Project $\mathrm{ECHO}$ is a powerful telehealth model providing mentorship and education to clinicians, encouraging them to treat more complex cases in their primary care clinics. As a result, patients receive higher quality care when they need it, and close to home, particularly important in rural areas.

4032

An exploration of the perceptions of young women with breast cancer with varying health literacy levels about the usefulness of cancer educational materials

Pearman Parker ${ }^{1}$, Jean C McSweeney ${ }^{1}$, Kristin Zorn ${ }^{2}$, Kristie Hadden $^{2}$, Carolyn Greene ${ }^{2}$, L. Joseph $\mathrm{Su}^{2}$, and Cathy Meade ${ }^{3}$ ${ }^{1}$ University of Arkansas Translational Research Institute; ${ }^{2}$ University of Arkansas for Medical Sciences; ${ }^{3}$ Moffitt Cancer Center

OBJECTIVES/GOALS: Young women (18 - 45 years of age) with breast cancer have complex medical and psychosocial needs. Educational materials are often used as tools in patient-centered communication. However, these materials disseminate complex health information in print-heavy formats and can be difficult to understand for women with varying health literacy levels. METHODS/STUDY POPULATION: In the first phase of this study, the principal investigator (PI) will recruit 40 diverse women to participate in four focus groups (FG) to explore the perceived usefulness of the most frequently used cancer educational materials. The PI will also obtain demographics and heath literacy levels of the FG participants using the Newest Vital Sign. In the second phase, the PI will assess the literacy demands of the ten most frequently used cancer educational print materials and five most frequently used websites described by the FG participants. The perceptions of the usefulness of materials and the literacy demands will then be used to appraise the effectiveness of materials within patient-centered cancer communication. RESULTS/ANTICIPATED RESULTS: Results from this study will provide a patient-centered blueprint that will be used to design more effective educational materials that treatment centers can incorporate into their patient-centered cancer communication process. The next step of this research will be to determine providers' perceptions of cancer education materials used to exchange information within the patient-centered communication process. This will complement the 